\title{
The Nyström Method and Convergence Analysis for System of Fredholm Integral Equations
}

\author{
Huimin Zhou ${ }^{1}$ and Qisheng Wang ${ }^{1 *}$ \\ ${ }^{1}$ School of Mathematics and Computational Science, Wuyi University, Jiangmen, Guangdong, P.R. China \\ *Corresponding author E-mail: 282228006@qq.com
}

\section{Article Info}

Keywords: Convergence analysis, Error estimation, Nyström method, System of Fredholm integral equations. 2010 AMS: 45D05, 45G10, 65D30.

Received: 23 November 2018

Accepted: 1 March 2019

Available online: 17 June 2019

\begin{abstract}
In this paper, the efficient numerical solutions of a class of system of Fredholm integral equations are solved by the Nyström method, which discretizes the system of integral equations into solving a linear system. The existence and uniqueness of the exact solutions are proved by the Banach fixed point theorem. The format of the Nyström solutions is given, especially with the composite Trapezoidal and Simpson rules. The results of error estimation and convergence analysis are obtained in the infinite norm sense. The validity and reliability of the theoretical analysis are verified by numerical experiments.
\end{abstract}

\section{Introduction}

In this paper, we consider a class of system of Fredholm integral equations of the form

$$
\left\{\begin{array}{l}
u(x)=f(x)+\int_{a}^{b}\left[k_{11}(x, y) u(y)+k_{12}(x, y) v(y)\right] d y \\
v(x)=g(x)+\int_{a}^{b}\left[k_{21}(x, y) u(y)+k_{22}(x, y) v(y)\right] d y,
\end{array}\right.
$$

where the known functions $f(x), g(x) \in C[a, b], k_{p q}(x, y) \in C([a, b] \times[a, b]), p, q=1,2, u(x), v(x) \in C[a, b]$ are the unknown functions. The integral equation problem has been two hundred years old, and the integral equation is widely used in the study of physics, especially in mechanics, magnetism, architecture and etc. Since the exact solution of the integral equation problem is difficult to find, its high-precision numerical solutions are often studied. Many numerical methods are used for numerical solution of Fredholm integral equation, for instance, Taylor collocation method [1], Galerkin projection and Least squares approximation method [2], variational iteration and fixed point iterative method [3], Nyström method and mechanical quadrature method [4]-[7], meshless methods [8] and multiscale methods [9], and so on. However, there is not much paper about solving the system of integral equations. This paper will study the Nyström method of the system of Fredholm integral equations.

\section{A sufficient condition for the existence and uniqueness of exact solutions}

According to Banach fixed point theorem, a sufficient condition for the existence and uniqueness of exact solution of system of Fredholm integral equations (1.1) is proposed. First, for (1.1), we structure a function vector space

$$
V^{2}[a, b]=\left\{s(x)=\left[s_{1}(x), s_{2}(x)\right]^{T}, s_{i}(x) \in C[a, b], \quad i=1,2\right\}
$$

and a functional matrix space

$$
V^{2 \times 2}([a, b] \times[a, b])=\left\{\left(s_{i j}(x, y)\right)_{2 \times 2}, s_{i j}(x, y) \in C([a, b] \times[a, b]), i, j=1,2\right\} .
$$

For

$$
K(x, y)=\left(k_{p q}(x, y)\right)_{2 \times 2} \in V^{2 \times 2}([a, b] \times[a, b]), \quad p, q=1,2
$$


and

$$
U(x)=[u(x), v(x)]^{T} \in V^{2}[a, b],
$$

we write the numerical integral operator $\mathscr{K}$ defined as

$$
(\mathscr{K} U)(x)=\int_{a}^{b} K(x, y) U(y) d y .
$$

The norm of the numerical integral operator $\mathscr{K}$ discussed in this paper is defined as

$$
\|\mathscr{K}\|_{\infty}=\max _{1 \leq p \leq 2}\left[\sum_{q=1}^{2} \max _{a \leq x \leq b} \int_{a}^{b}\left|k_{p q}(x, y)\right| d y\right] .
$$

Theorem 2.1. If $\|\mathscr{K}\|_{\infty}<1$ holds, then the exact solutions of the system of Fredholm integral equations (1.1) is existential and unique.

Proof. For all $U_{i}(x) \in V^{2}[a, b], i=1,2$, one has

$$
T U_{i}=F(x)+\int_{a}^{b} K(x, y) U(y) d y, \quad i=1,2
$$

Then we have

$$
\begin{aligned}
\left\|T U_{1}-T U_{2}\right\|_{\infty} & =\left\|\int_{a}^{b} K(x, y) U_{1}(y) d y-\int_{a}^{b} K(x, y) U_{2}(y) d y\right\|_{\infty} \\
& =\left\|\int_{a}^{b} K(x, y)\left[U_{1}(y)-U_{2}(y)\right] d y\right\|_{\infty} \\
& \leq\left\|\int_{a}^{b} K(x, y) d y\right\|_{\infty} \cdot\left\|U_{1}-U_{2}\right\|_{\infty} \\
& \leq\|\mathscr{K}\|_{\infty} \cdot\left\|U_{1}-U_{2}\right\|_{\infty} .
\end{aligned}
$$

Since $\|\mathscr{K}\|_{\infty}<1, T$ is a contraction mapping. Consider that Banach fixed point theorem, then (1.1) exists a unique solution $U^{*} \in V^{2}[a, b]$ such that $T U^{*}=U^{*}$ holds.

\section{The Nyström method}

In this section, we use the numerical quadrature scheme to obtain a general algorithm for the Nyström method of the system of Fredholm integral equations.

Applying numerical quadrature scheme to integral terms of (1.1), we can have

$$
\begin{aligned}
& \int_{a}^{b}\left[k_{p 1}(x, y) u(y)+k_{p 2}(x, y) v(y)\right] d y \\
& =\sum_{i=0}^{n} \omega_{i}\left[k_{p 1}\left(x, x_{i}\right) u\left(x_{i}\right)+k_{p 2}\left(x, x_{i}\right) v\left(x_{i}\right)\right]+R_{p}^{(n)}, \quad p=1,2,
\end{aligned}
$$

where $\omega_{i}(i=0,1, \ldots, n)$ are coefficients of quadrature and $x_{i}(i=0,1, \ldots, n)$ are the quadrature node points and $R_{1}^{(n)}, R_{2}^{(n)}$ are remainder terms, such that (1.1) can be rewritten as

$$
\left\{\begin{array}{l}
u(x)=f(x)+\sum_{i=0}^{n} \omega_{i}\left[k_{11}\left(x, x_{i}\right) u\left(x_{i}\right)+k_{12}\left(x, x_{i}\right) v\left(x_{i}\right)\right]+R_{1}^{(n)} \\
v(x)=g(x)+\sum_{i=0}^{n} \omega_{i}\left[k_{21}\left(x, x_{i}\right) u\left(x_{i}\right)+k_{22}\left(x, x_{i}\right) v\left(x_{i}\right)\right]+R_{2}^{(n)} .
\end{array}\right.
$$

We take the collocation points $x=x_{i}$, and let $f\left(x_{i}\right)=f_{i}, g\left(x_{i}\right)=g_{i}, k_{p q}\left(x_{i}, x_{j}\right)=k_{p q}^{i j}, u\left(x_{i}\right)=u_{i}, v\left(x_{i}\right)=v_{i}, i=0,1, \ldots, n ; p, q=1,2$.

Then we ignore the remainder terms and obtain the approximating linear system with respect to $u_{0}, v_{0}, u_{1}, v_{1}, \ldots, u_{n}, v_{n}$ as

$$
\left\{\begin{array}{l}
u_{i}=f_{i}+\sum_{j=0}^{n} \omega_{j}\left(k_{11}^{i j} u_{j}+k_{12}^{i j} v_{j}\right) \\
v_{i}=g_{i}+\sum_{j=0}^{n} \omega_{j}\left(k_{21}^{i j} u_{j}+k_{22}^{i j} v_{j}\right)
\end{array}\right.
$$

Remove the terms of (3.2), then we obtain

$$
\left\{\begin{array}{l}
-\sum_{j \neq i} \omega_{j} k_{11}^{i j} u_{j}+\left(1-\omega_{i} k_{11}^{i i}\right) u_{i}-\sum_{j=0}^{n} \omega_{j} k_{12}^{i j} v_{j}=f_{i} \\
-\sum_{j=0}^{n} \omega_{j} k_{21}^{i j} u_{j}-\sum_{j \neq i} \omega_{j} k_{22}^{i j} v_{j}+\left(1-\omega_{i} k_{22}^{i i}\right) v_{i}=g_{i}
\end{array}\right.
$$

Solve linear system (3.3), we can get $u_{i}=u_{i}^{*}, v_{i}=v_{i}^{*}, i=0,1, \ldots, n$.

Take $u_{i}^{*}, v_{i}^{*}$ into (3.1) omitting the remainder terms, we have

$$
\left\{\begin{array}{l}
u_{n}(x)=f(x)+\sum_{i=0}^{n} \omega_{i}\left[k_{11}\left(x, x_{i}\right) u_{i}^{*}+k_{12}\left(x, x_{i}\right) v_{i}^{*}\right] \\
v_{n}(x)=g(x)+\sum_{i=0}^{n} \omega_{i}\left[k_{21}\left(x, x_{i}\right) u_{i}^{*}+k_{22}\left(x, x_{i}\right) v_{i}^{*}\right]
\end{array}\right.
$$

Thus $u_{n}(x), v_{n}(x)$ can be called the Nyström solutions with numerical quadrature scheme (3.1). Meanwhile, it can be noted that

$$
\left\{\begin{array}{l}
u_{n}\left(x_{i}\right)=u_{i}^{*} \\
v_{n}\left(x_{i}\right)=v_{i}^{*}
\end{array}\right.
$$

so $u_{n}(x), v_{n}(x)$ are also the Nyström interpolation functions under the interpolation condition (3.2). 


\section{Error estimation}

To carry out an error analysis for the Nyström method, we first give the following useful Lemma.

Lemma 4.1. Let $a_{1}, a_{2}, b_{1}, b_{2}, c_{1}, c_{2}$, and $x, y$ are positive real numbers. Assume

$$
\left\{\begin{array}{l}
x \leq a_{1}+b_{1} x+c_{1} y \\
y \leq a_{2}+b_{2} x+c_{2} y
\end{array}\right.
$$

For $b_{1}+c_{2}<1$ and $\left(1-b_{1}\right)\left(1-c_{2}\right)>b_{2} c_{1}$, then

$$
x+y \leq \frac{\left(1+b_{2}-c_{2}\right) a_{1}+\left(1+c_{1}-b_{1}\right) a_{2}}{\left(1-b_{1}\right)\left(1-c_{2}\right)-b_{2} c_{1}} .
$$

The proof of this Lemma can be given directly and we omit it. The result of error estimation is given below.

Theorem 4.2. Let $u(x), v(x)$ are the exact solutions and let $u_{n}(x), v_{n}(x)$ are the Nyström solutions of system of Fredholm integral equations (1.1). Assume $M_{11}+M_{22}<1$, and $\left(1-M_{11}\right)\left(1-M_{22}\right)>M_{12} M_{21}$, then

$$
\left\|u-u_{n}\right\|_{\infty}+\left\|v-v_{n}\right\|_{\infty} \leq \frac{\left(1-M_{22}+M_{21}\right)\left\|R_{1}^{(n)}\right\|_{\infty}+\left(1-M_{11}+M_{12}\right)\left\|R_{2}^{(n)}\right\|_{\infty}}{\left(1-M_{11}\right)\left(1-M_{22}\right)-M_{21} M_{12}},
$$

where $M_{p q}=(b-a)\left\|k_{p q}(x, y)\right\|_{\infty}, \quad p, q=1,2$.

Proof. Consider (3.5) and subtract (3.4) from (3.1) to get

$$
\begin{aligned}
u-u_{n} & =\sum_{i=0}^{n} \omega_{i}\left[k_{11}\left(x, x_{i}\right)\left(u\left(x_{i}\right)-u_{i}^{*}\right)+k_{12}\left(x, x_{i}\right)\left(v\left(x_{i}\right)-v_{i}^{*}\right)\right]+R_{1}^{(n)} \\
& =\sum_{i=0}^{n} \omega_{i}\left[k_{11}\left(x, x_{i}\right)\left(u\left(x_{i}\right)-u_{n}\left(x_{i}\right)\right)+k_{12}\left(x, x_{i}\right)\left(v\left(x_{i}\right)-v_{n}\left(x_{i}\right)\right)\right]+R_{1}^{(n)},
\end{aligned}
$$

then

$$
\left\|u-u_{n}\right\|_{\infty} \leq\left\|R_{1}^{(n)}\right\|_{\infty}+\left\|\sum_{i=0}^{n}\left|\omega_{i} k_{11}\left(x, x_{i}\right)\right|\right\|_{\infty} \cdot\left\|u-u_{n}\right\|_{\infty}+\left\|\sum_{i=0}^{n}\left|\omega_{i} k_{12}\left(x, x_{i}\right)\right|\right\|_{\infty} \cdot\left\|v-v_{n}\right\|_{\infty} .
$$

Similarly, we have

$$
\left\|v-v_{n}\right\|_{\infty} \leq\left\|R_{2}^{(n)}\right\|_{\infty}+\left\|\sum_{i=0}^{n}\left|\omega_{i} k_{21}\left(x, x_{i}\right)\right|\right\|_{\infty} \cdot\left\|u-u_{n}\right\|_{\infty}+\left\|\sum_{i=0}^{n}\left|\omega_{i} k_{22}\left(x, x_{i}\right)\right|\right\|_{\infty} \cdot\left\|v-v_{n}\right\|_{\infty} .
$$

From the intermediate value theorem of continuous function, we can get

$$
\begin{aligned}
\left\|\sum_{i=0}^{n}\left|\omega_{i} k_{p q}\left(x, x_{i}\right)\right|\right\|_{\infty} & =(b-a)\left\|k_{p q}\left(x, \eta_{p q}\right) \mid\right\|_{\infty} \\
& \leq(b-a)\left\|k_{p q}(x, y) \mid\right\|_{\infty}
\end{aligned}
$$

where $\eta_{p q} \in[a, b], \quad p, q=1,2$.

It follows that a system of inequalities

$$
\left\{\begin{array}{l}
\left\|u-u_{n}\right\|_{\infty} \leq\left\|R_{1}^{(n)}\right\|_{\infty}+M_{11}\left\|u-u_{n}\right\|_{\infty}+M_{12}\left\|v-v_{n}\right\|_{\infty} \\
\left\|v-v_{n}\right\|_{\infty} \leq\left\|R_{2}^{(n)}\right\|_{\infty}+M_{21}\left\|u-u_{n}\right\|_{\infty}+M_{22}\left\|v-v_{n}\right\|_{\infty}
\end{array}\right.
$$

From Lemma 4.1, we can obtain

$$
\left\{\begin{aligned}
\left\|u-u_{n}\right\|_{\infty} \leq \frac{\left(1-M_{22}\right)\left\|R_{1}^{(n)}\right\|_{\infty}+M_{12}\left\|R_{2}^{(n)}\right\|_{\infty}}{\left(1-M_{11}\right)\left(1-M_{22}\right)-M_{21} M_{12}} \\
\left\|v-v_{n}\right\|_{\infty} \leq \frac{M_{21}\left\|R_{1}^{(n)}\right\|_{\infty}+\left(1-M_{11}\right)\left\|R_{2}^{(n)}\right\|_{\infty}}{\left(1-M_{11}\right)\left(1-M_{22}\right)-M_{21} M_{12}}
\end{aligned}\right.
$$

when $M_{11}+M_{22}<1$ and $\left(1-M_{11}\right)\left(1-M_{22}\right)>M_{21} M_{12}$.

In particular, for the composite trapezoidal rules, we have

$$
\left\{\begin{array}{l}
h=\frac{b-a}{n} \\
\omega_{0}=\omega_{n}=\frac{h}{2} \\
\omega_{1}=\omega_{2}=\ldots=\omega_{n-1}=h \\
x_{i}=x_{0}+n h, \quad i=0,1, \ldots, n
\end{array}\right.
$$

so

$$
\begin{aligned}
\sum_{i=0}^{n}\left|\omega_{i} k_{p q}\left(x, x_{i}\right)\right| & =\frac{b-a}{n}\left[\frac{1}{2}\left(\left|k_{p q}\left(x, x_{0}\right)\right|+\left|k_{p q}\left(x, x_{n}\right)\right|\right)+\sum_{i=1}^{n-1}\left|k_{p q}\left(x, x_{i}\right)\right|\right] \\
& =(b-a)\left|k_{p q}\left(x, \eta_{p q}\right)\right|, \quad \eta_{p q} \in[a, b] .
\end{aligned}
$$

Conseqently, $\left\|\sum_{i=0}^{n}\left|\omega_{i} k_{p q}\left(x, x_{i}\right)\right|\right\|_{\infty}=M_{p q}, \quad p, q=1,2$.

Let

$$
\left\{\begin{array}{l}
k_{1}(x, \xi)=\frac{\partial^{2}}{\partial y^{2}}\left[k_{11}(x, y) u(y)+k_{12}(x, y) v(y)\right]_{y=\xi}, a<\xi<b \\
k_{2}(x, \eta)=\frac{\partial^{2}}{\partial y^{2}}\left[k_{21}(x, y) u(y)+k_{22}(x, y) v(y)\right]_{y=\eta}, a<\eta<b .
\end{array}\right.
$$

To sum up, we can draw the following corollary. 
Corollary 4.3. If $M_{11}+M_{22}<1$ and $\left(1-M_{11}\right)\left(1-M_{22}\right)>M_{12} M_{21}$, then the Nyström solutions with the composite trapezoidal quadrature formula have the following error estimation:

$$
\left\|u-u_{n}\right\|_{\infty}+\left\|v-v_{n}\right\|_{\infty} \leq \frac{b-a}{12} \frac{\left(1-M_{22}+M_{21}\right)\left\|k_{1}(x, y)\right\|_{\infty}+\left(1-M_{11}+M_{12}\right)\left\|k_{2}(x, y)\right\|_{\infty}}{\left(1-M_{11}\right)\left(1-M_{22}\right)-M_{21} M_{12}} h^{2} .
$$

By a similar argument, for the composite Simpson rules, we have

$$
\left\{\begin{array}{l}
h=\frac{b-a}{n} \\
\omega_{0}=\omega_{2 n}=\frac{h}{6} \\
\omega_{1}=\omega_{3}=\ldots=\omega_{2 n-1}=\frac{2}{3} h \\
\omega_{2}=\omega_{4}=\ldots=\omega_{2 n-2}=\frac{1}{3} h \\
x_{i}=x_{0}+i \frac{h}{2}, \quad i=0,1, \ldots, 2 n
\end{array}\right.
$$

hence

$$
\begin{aligned}
\sum_{i=0}^{2 n}\left|\omega_{i} k_{p q}\left(x, x_{i}\right)\right| & =\frac{b-a}{n}\left[\frac{1}{6}\left(\left|k_{p q}\left(x, x_{0}\right)+k_{p q}\left(x, x_{2 n}\right)\right|\right)+\frac{1}{3} \sum_{i=1}^{n-1}\left|k_{p q}\left(x, x_{2 i}\right)\right|+\frac{2}{3} \sum_{i=1}^{n}\left|k_{p q}\left(x, x_{2 i-1}\right)\right|\right] \\
& =(b-a) k_{p q}\left(x, \eta_{p q}\right), \quad \eta_{p q} \in[a, b] .
\end{aligned}
$$

Then $\left\|\sum_{i=0}^{2 n} \mid \omega_{i} k_{p q}\left(x, x_{i}\right)\right\|_{\infty}=M_{p q}, \quad p, q=1,2$.

Let

$$
\left\{\begin{array}{l}
\bar{k}_{1}(x, \xi)=\frac{\partial^{4}}{\partial y^{4}}\left[k_{11}(x, y) u(y)+k_{12}(x, y) v(y)\right]_{y=\xi}, a<\xi<b \\
\bar{k}_{2}(x, \eta)=\frac{\partial^{4}}{\partial y^{4}}\left[k_{21}(x, y) u(y)+k_{22}(x, y) v(y)\right]_{y=\eta}, a<\eta<b .
\end{array}\right.
$$

Again, we can draw the following corollary.

Corollary 4.4. If $M_{11}+M_{22}<1$ and $\left(1-M_{11}\right)\left(1-M_{22}\right)>M_{12} M_{21}$, then the Nyström solutions with the composite Simpson quadrature formula have the following error estimation:

$$
\left\|u-u_{n}\right\|_{\infty}+\left\|v-v_{n}\right\|_{\infty} \leq \frac{b-a}{180} \frac{\left(1-M_{22}+M_{21}\right)\left\|\bar{k}_{1}(x, y)\right\|_{\infty}+\left(1-M_{11}+M_{12}\right)\left\|\bar{k}_{2}(x, y)\right\|_{\infty}}{\left(1-M_{11}\right)\left(1-M_{22}\right)-M_{21} M_{12}}\left(\frac{h}{2}\right)^{4} .
$$

\section{Numerical examples}

In order to verify the validity of the proposed numerical method, two numerical examples are given and the exact solutions are compared with the approximate solutions by using Matlab.R2015a. The convergence rate is defined by

$$
\text { Ratio }=\frac{\left\|u-u_{n}\right\|_{\infty}+\left\|v-v_{n}\right\|_{\infty}}{\left\|u-u_{2 n}\right\|_{\infty}+\left\|v-v_{2 n}\right\|_{\infty}} .
$$

Example 5.1. Consider the following system of Fredholm integral equations

$$
\left\{\begin{array}{l}
u(x)=x^{2}+\frac{5}{24} x-\frac{7}{24}+\int_{0}^{1}\left[\left(-\frac{1}{2} x+\frac{1}{2} y\right) u(y)+\left(\frac{1}{12} x-y\right) v(y)\right] d y \\
v(x)=\frac{7}{9} x-\frac{19}{18}+\int_{0}^{1}\left[x y u(y)+\frac{1}{6}(x y-2 y) v(y)\right] d y
\end{array}\right.
$$

with $0 \leq x \leq 1$ and the exact solutions $u(x)=x^{2}, v(x)=x-1$.

We choose $n=4,8,16,32$ along with $h=\frac{1}{n}$ and get $x_{i}=i h, \quad i=0,1, \ldots, n$. The curve graph of the exact solutions $u(x)=x^{2}, v(x)=$ $x-1$ and the approximations $u_{n}(x), v_{n}(x)$ obtained using the Nyström method are given in Figure 5.1(a), and then the maximum error $\left\|u-u_{n}\right\|_{\infty}+\left\|v-v_{n}\right\|_{\infty}$ listed in Table 1 .

\begin{tabular}{|c|c|c|c|c|}
\hline & \multicolumn{2}{|c|}{ Composite trapezoidal } & \multicolumn{2}{c|}{ Composite Simpson } \\
\hline$n$ & $\left\|u-u_{n}\right\|_{\infty}+\left\|v-v_{n}\right\|_{\infty}$ & Ratio & $\left\|u-u_{n}\right\|_{\infty}+\left\|v-v_{n}\right\|_{\infty}$ & Ratio \\
\hline 4 & $1.7800 \mathrm{e}-02$ & & 0 & \\
\hline 8 & $4.5000 \mathrm{e}-03$ & 3.9556 & 0 & 0 \\
\hline 16 & $1.1000 \mathrm{e}-03$ & 4.0909 & $5.7465 \mathrm{e}-19$ & 0 \\
\hline 32 & $2.7778 \mathrm{e}-04$ & 3.9600 & 0 & 0 \\
\hline
\end{tabular}

Table 1: Error calculation result of Example 5.1.

Example 5.2. Consider the following system of Fredholm integral equations

$$
\left\{\begin{array}{l}
u(x)=\sin x+\int_{0}^{2 \pi}\left[\left(\frac{1}{20} \sin x-\cos y\right) u(y)+\left(\frac{1}{40} y \sin x\right) v(y)\right] d y \\
v(x)=\cos x-\frac{11 \pi}{20}+\int_{0}^{2 \pi}\left[\left(\frac{1}{50} \sin x-\frac{1}{40} y\right) u(y)+\left(\frac{1}{18} \sin x+\frac{1}{2} \cos y\right) v(y)\right] d y,
\end{array}\right.
$$

with $0 \leq x \leq 2 \pi$ and the exact solutions $u(x)=\sin x, v(x)=\cos x$.

We also choose $n=4,8,16,32$ along with $h=\frac{1}{n}$ and get $x_{i}=i h, \quad i=0,1, \ldots, n$. The curve graph of the exact solutions $u(x)=\sin x$, $v(x)=\cos x$ and the approximations $u_{n}(x), v_{n}(x)$ obtained using the Nyström method are given in Figure 5.1(b), and then the maximum errors $\left\|u-u_{n}\right\|_{\infty}+\left\|v-v_{n}\right\|_{\infty}$ listed in Table 2 . 


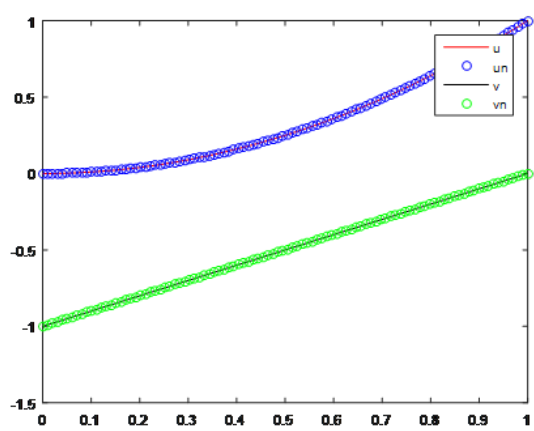

(a) Example 5.1

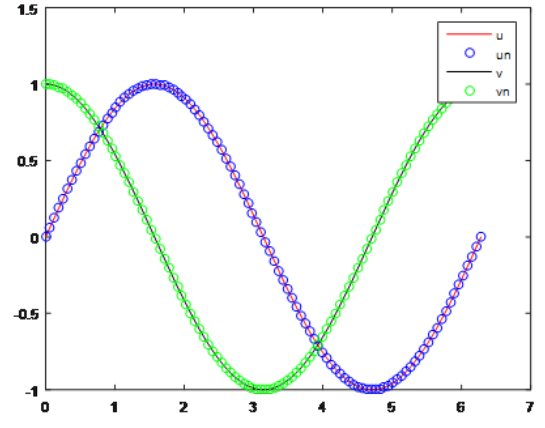

(b) Example 5.2

Figure 5.1: The exact solutions and the Nyström solutions of Example 5.1 and Example 5.2 when $n=16$

\begin{tabular}{|c|c|c|c|c|}
\hline & \multicolumn{2}{|c|}{ Composite trapezoidal } & \multicolumn{2}{c|}{ Composite Simpson } \\
\hline$n$ & $\left\|u-u_{n}\right\|_{\infty}+\left\|v-v_{n}\right\|_{\infty}$ & Ratio & $\left\|u-u_{n}\right\|_{\infty}+\left\|v-v_{n}\right\|_{\infty}$ & Ratio \\
\hline 4 & $6.4300 \mathrm{e}-02$ & & $6.8765 \mathrm{e}-04$ & \\
\hline 8 & $1.5700 \mathrm{e}-02$ & 4.0955 & $4.0590 \mathrm{e}-05$ & 16.9414 \\
\hline 16 & $3.9000 \mathrm{e}-03$ & 4.0256 & $2.5019 \mathrm{e}-06$ & 16.2237 \\
\hline 32 & $9.6947 \mathrm{e}-04$ & 4.0228 & $1.5583 \mathrm{e}-07$ & 16.0553 \\
\hline
\end{tabular}

Table 2: Error calculation result of Example 5.2

\section{Conclusion}

In this paper, The Nyström method is proposed to handle approximate solutions of system of Fredholm integral equations and two numerical examples are provided to illustrate the validity and feasibility of the present method. For the simple system of integral equations such as polynomial integral equations, we appear to get the exact solutions directly by the Nyström method with the composite Simpson rule. In the future, the Nyström method can be extended to solve Hammerstein integral equations. A two-grid iteration method for the Nyström method for system of Fredholm integral equations will also be further studied.

\section{Acknowledgement}

This research was supported by the Natural Science Foundation of Guangdong Province(Grant No.2015A030313643,2016A030313005), Postgraduate Demonstration Course Construction Project of Guangdong Province(Grant No.2018SFKC38) and Postgraduate Demonstration Course Construction Project of Wuyi University(Grant No.YJS-SFKC-18-01).

\section{References}

[1] K. Y. Wang, Q. S. Wang, Taylor collocation method and convergence analysis for the Volterra-Fredholm integral equations, J. Comput. Appl. Math., 260 (2014), 294-300.

[2] Q. S. Wang, K. Y. Wang, S. J. Chen, Least squares approximation method for the solution of Volterra-Fredholm integral equations, J. Comput. Appl. Math., 272 (2014), 141-147.

[3] K. Y. Wang, Q. S. Wang, K. Z. Guan, Iterative method and convergence analysis for a kind of mixed nonlinear Volterra-Fredholm integral equation, Appl. Math. Comput., 225 (2013), 631-637.

[4] K. Atkinson, W. Han, Theoretical Numerical Analysis: A Functional Analysis Framework (Third Edition), Springer, 2009.

[5] X. C. Zhong, A new Nyström-type method for Fredholm integral equations of the second kind, Appl. Math. Comput., 219 (2013), $8842-8847$.

[6] L. J. Lardy, A Variation of Nyström's Method for Hammerstein Equations, J. Integral. Equ., 3(1) (1981), 43-60.

[7] J. Dick, P. Kritzer, F. Y. Kuo, I. H. Sloan, Lattice-Nyström method for Fredholm integral equations of the second kind with convolution type kernels, J. Complexity., 23 (2007), 752-772.

[8] Q. S. Wang, H. S. Wang, Meshless method and convergence analysis for 2-dimensional Fredholm integral equation with complex factors, J. Comput. Appl. Math., 304 (2016), 18-25.

[9] Z. Y. Chen, C. A. Micchelli, Y. S. Xu, Multiscale Methods for Fredholm Integral Equations, Cambridge University Press, 2015. 